\title{
Cardiomyopathy-Related Mutations in Cardiac Troponin C, L29Q and G159D, Have Divergent Effects on Rat Cardiac Myofiber Contractile Dynamics
}

\author{
Sampath K. Gollapudi and Murali Chandra \\ Department of Veterinary and Comparative Anatomy, Pharmacology, and Physiology (VCAPP), \\ Washington State University, Pullman, WA 99164-6520, USA \\ Correspondence should be addressed to Sampath K. Gollapudi, sampath@vetmed.wsu.edu
}

Received 14 May 2012; Revised 6 July 2012; Accepted 8 August 2012

Academic Editor: Danuta Szczesna-Cordary

Copyright (C 2012 S. K. Gollapudi and M. Chandra. This is an open access article distributed under the Creative Commons Attribution License, which permits unrestricted use, distribution, and reproduction in any medium, provided the original work is properly cited.

\begin{abstract}
Previous studies of cardiomyopathy-related mutations in cardiac troponin C (cTnC) - L29Q and G159D-have shown diverse findings. The link between such mutant effects and their divergent impact on cardiac phenotypes has remained elusive due to lack of studies on contractile dynamics. We hypothesized that a cTnC mutant-induced change in the thin filament will affect global myofilament mechanodynamics because of the interactions of thin filament kinetics with both $\mathrm{Ca}^{2+}$ binding and crossbridge (XB) cycling kinetics. We measured pCa-tension relationship and contractile dynamics in detergent-skinned rat cardiac papillary muscle fibers reconstituted with the recombinant wild-type rat $c \operatorname{TnC}\left(\mathrm{cTnC}_{\mathrm{WT}}\right), \mathrm{cTnC} \mathrm{C}_{\mathrm{L} 29 \mathrm{Q}}$, and $c \mathrm{TnC}_{\mathrm{G} 159 \mathrm{D}}$ mutants. cTnC $\mathrm{C}_{\mathrm{L} 29 \mathrm{Q}}$ fibers demonstrated a significant decrease in $\mathrm{Ca}^{2+}$ sensitivity, but $\mathrm{cTnC}_{\mathrm{G} 159 \mathrm{D}}$ fibers did not. Both mutants had no effect on $\mathrm{Ca}^{2+}$-activated maximal tension. The rate of $\mathrm{XB}$ recruitment dynamics increased in $\mathrm{cTnC}_{\mathrm{L} 29 \mathrm{Q}}(26 \%)$ and $\mathrm{cTnC}_{\mathrm{G} 159 \mathrm{D}}(25 \%)$ fibers. The rate of $\mathrm{XB}$ distortion dynamics increased in $\mathrm{cTnC}_{\mathrm{G} 159 \mathrm{D}}$ fibers $(15 \%)$. Thus, the $\mathrm{CTnC}_{\mathrm{L} 29 \mathrm{Q}}$ mutant modulates the equilibrium between the non-cycling and cycling pool of XB by affecting the on/off kinetics of the regulatory units (Tropomyosin-Troponin); whereas, the $\mathrm{cTnC}_{\mathrm{G} 159 \mathrm{D}}$ mutant increases XB cycling rate. Different effects on contractile dynamics may offer clue regarding how $\mathrm{cTnC}_{\mathrm{L} 29 \mathrm{Q}}$ and $\mathrm{cTnC}_{\mathrm{G159D}}$ cause divergent effects on cardiac phenotypes.
\end{abstract}

\section{Introduction}

The presumptive conclusion drawn from the heterogenic nature of human cardiomyopathy suggests a link between the type of mutation and the nature of pathological remodeling of the heart. A growing number of mutations in human cardiac troponin $\mathrm{C}(\mathrm{cTnC})$, associated with either hypertrophic cardiomyopathy (HCM) or dilated cardiomyopathy (DCM), makes it a prominent target gene for functional characterization. Thus far, 5 mutations in $\mathrm{cTnC}$ are found to be associated with HCM and 6 with DCM. These HCMlinked cTnC mutations include L29Q [1]-A8V, C84Y, and E134D [2]-Q122AfsX30 [3], while the DCM-linked cTnC mutations include E59D/D75Y [4], G159D [5]-Y5H, M103I, D145E, and I148V [6]. cTnC comprises two globular lobes; the amino- $(\mathrm{N})$ and the carboxyl- $(\mathrm{C})$ terminal lobes, which are connected by a flexible linker. The binding of $\mathrm{Ca}^{2+}$ to the regulatory site-II of the N-lobe of cTnC is important for triggering structural changes in the regulatory unit (RU), consisting of troponin ( $\mathrm{Tn}$ ) and tropomyosin (Tm).

The binding of $\mathrm{Ca}^{2+}$ to cTnC has a strong influence on the rates of transition between off and on states of RU (Tm$\mathrm{Tn})$ and the thin filament activation. Moreover, the on and off kinetic states of RU depend on XB in the force-bearing state through cooperative mechanisms $[7,8]$. Therefore, mutations in $\mathrm{cTnC}$ lead to the expectation that $\mathrm{Ca}^{2+}$ binding kinetics of thin filaments are altered. Two mutations in cTnC are of particular interest to our study: L29Q mutation in the N-lobe, and G159D mutation in the C-lobe. Because of its close proximity to site-II of $\mathrm{cTnC}$, leucine to glutamine substitution at position 29 (L29Q) is considered to have a direct effect on the $\mathrm{Ca}^{2+}$ binding properties of cTnC [911]. On the other hand, aspartic acid to glycine substitution at position 159 (G159D) is thought to affect interactions 
of cTnC with cardiac TnI (cTnI) and cardiac troponin T (cTnT), and possibly $\operatorname{Tm}[12,13]$.

The first mutation, L29Q, in cTnC was discovered in a 60-year old male, who was diagnosed with concentric hypertrophy in the left ventricle [1]. However, the patient showed no signs of diastolic and systolic dysfunction [1]. On the other hand, the proband of the G159D mutation was diagnosed with DCM at the age of 21 [5]. The proband displayed sudden heart failure symptoms and required a heart transplant two months after diagnosis [5]. Previous in vitro studies of L29Q and G159D mutations reported contrasting findings, making it difficult to correlate the functional effects observed to the known cardiac phenotypes. Functional studies on the L29Q mutant reported an increase [10], a decrease [11], or no change in the myofilament $\mathrm{Ca}^{2+}$ sensitivity $[14,15]$. Studies of G159D mutant also resulted in diverse findings on $\mathrm{Ca}^{2+}$ sensitivity; with one group reporting an increase [16], several groups reporting a decrease $[14,17,18]$, and other groups reporting no effect at all $[19,20]$. Such discrepancies may be primarily related to the use of heterologous tissue/proteins, extracted from pig, bovine, rabbit, human, rat, and mouse species. A cursory look at the proteins from these species reveal multiple amino acid differences, which render it difficult to use them in an assay that is designed to test the effect of a single amino acid exchange. Therefore, to permit an unambiguous understanding of the functional effects of point mutations in $c \operatorname{TnC}$, it is imperative to minimize heterogeneity in experimental conditions.

An important question is whether the effects of cTnC mutations go beyond that of previously observed mild-tomoderate changes in myofilament $\mathrm{Ca}^{2+}$ sensitivity $[10,11$, $14,16-18]$. Because of the interactions of thin filament kinetics with both $\mathrm{Ca}^{2+}$ binding and $\mathrm{XB}$ cycling kinetics, we predict that a cTnC mutant-induced effect on the thin filament would affect the other kinetic paradigms; the net effect is a change in the overall mechanodynamics of the whole myofilament system. Therefore, we hypothesized that a cTnC mutant-induced change will be expressed as a change in global myofilament mechanodynamics. To test our hypothesis, we studied the functional effects of L29Q and G159D cTnC mutations on contractile dynamics in detergent-skinned rat cardiac papillary muscle fibers reconstituted with homologous rat cardiac Tn subunits. L29Q substitution caused a small but significant decrease in $\mathrm{Ca}^{2+}$ sensitivity, while G159D mutation resulted in no effect. The rate constant that governs the length-mediated XB recruitment dynamics was faster in both L29Q and G159D mutants. The rate constant that describes the lengthmediated XB distortion dynamics was faster in the G159D mutant. We discuss these data in terms of cTnC mutantinduced effect on global myofilament mechanodynamics.

\section{Methods}

2.1. Preparation of Detergent-Skinned Cardiac Papillary Muscle Fiber Bundles. All animals used in this study received proper care and treatment in accordance with the guidelines set by the Washington State University Institutional
Animal Care and Use Committee. Papillary muscle fiber bundles from Sprague-Dawley rat hearts were prepared using the procedure described previously [21]. Briefly, rats were anaesthetized using isoflurane until they were deeply sedated; the depth of anesthesia was confirmed using a pedal withdrawal reflex. Hearts were quickly excised and placed into an ice-cold relaxing solution of pCa 9.0 [22]. Papillary bundles were removed from left ventricles of rat hearts and were further dissected into thinner bundles measuring 2.0$3.0 \mathrm{~mm}$ in length and $150-200 \mu \mathrm{m}$ in cross-section. Thinner fiber bundles were detergent-skinned overnight in relaxing solution that contained $1 \%$ Triton-X-100 [23].

\subsection{Expression and Purification of Recombinant Rat Cardiac} Tn Subunits. Recombinant $c$-myc tagged rat cardiac troponin $\mathrm{T}$ ( $c-m y c \mathrm{RcTnT}$ ), troponin I (RcTnI), and troponin $\mathrm{C}(\mathrm{RcTnC})$, were all cloned into a pSBETa vector, and were expressed in $\mathrm{BL} 21 * \mathrm{DE} 3$ cells (Novagen, Madison, WI) for protein synthesis. L29Q and G159D substitutions in RcTnC were generated using site-directed DNA mutagenesis techniques and were cloned into a pSBETa vector. BL21 $*$ DE3 cells were lysed and the proteins ( $c-m y c$ RcTnT, RcTnI, and $\mathrm{Rc} \operatorname{TnC}$ ) were purified using ion-exchange chromatography techniques, as described previously $[22,24,25]$. In brief, $\mathrm{BL} 21 * \mathrm{DE} 3$ cells of each protein preparation ( $\sim 4$ liters) were spun down and sonicated in $50 \mathrm{mM}$ Tris $\left(\mathrm{pH} 8.0\right.$ at $\left.4^{\circ} \mathrm{C}\right)$, $6 \mathrm{M}$ urea, $5 \mathrm{mM}$ EDTA, $0.2 \mathrm{mM}$ PMSF, $5 \mathrm{mM}$ benzamidine$\mathrm{HCl}, 10 \mathrm{mM}$ leupeptin, $1 \mathrm{mM}$ pepstatin, $5 \mathrm{mM}$ bestatin, $2 \mathrm{mM} \mathrm{E}-64$, and $1 \mathrm{mM}$ DTT. The insoluble fraction in each preparation was removed by centrifugation. $c-m y c$ RcTnT was purified by fractionation of the supernatant from the culture preparation using ammonium sulfate. The pellet from the $70 \%$ ammonium sulfate cut was first dissolved in $50 \mathrm{mM}$ Tris ( $\mathrm{pH} 8.0$ at $4^{\circ} \mathrm{C}$ ), $6 \mathrm{M}$ urea, $1 \mathrm{mM}$ EDTA, $0.2 \mathrm{mM}$ PMSF, $4 \mathrm{mM}$ benzamidine- $\mathrm{HCl}$, and $1 \mathrm{mM}$ DTT, and then purified by chromatography on a DEAE-fast Sepharose column [22]. RcTnT was eluted from the column using a linear $\mathrm{NaCl}$ gradient. RcTnI was purified by directly loading the supernatant from the RcTnI culture preparation onto a $\mathrm{CM}$ cation-exchange column and eluted using a linear $\mathrm{NaCl}$ gradient. Complete details on cTnI purification can be found in the study by Guo et al. [24]. Wild-type (WT) RcTnC, RcTnC-L29Q, and RcTnC-G159D were purified by loading the supernatant from each RcTnC culture preparation onto a DE-52 anion-exchange column and were eluted using a linear $\mathrm{KCl}$ gradient [25]. More details on the purification of cTnC can be found in the study by Pan and Johnson [25]. All fractions containing pure proteins were pooled and dialyzed thoroughly against deionized water containing $15 \mathrm{mM} \beta$ mercaptoethanol. Dialyzed proteins were lyophilized and stored at $-80^{\circ} \mathrm{C}$.

2.3. Reconstitution of Recombinant Rat Cardiac Tn Subunits into Detergent-Skinned Rat Cardiac Muscle Fiber Bundles. The reconstitution of recombinant cardiac Tn subunits into muscle fiber bundles was performed using a protocol described previously [22]. Briefly, we first prepared an extraction solution containing RcTnT-RcTnI by dissolving c-myc RcTnT $(1.5 \mathrm{mg} / \mathrm{mL}, \mathrm{W} / \mathrm{V})$ and RcTnI $(1.0 \mathrm{mg} / \mathrm{mL}$, 
$\mathrm{W} / \mathrm{V})$ in $50 \mathrm{mM}$ Tris- $\mathrm{HCl}(\mathrm{pH} 8.0), 6 \mathrm{M}$ urea, $1.0 \mathrm{M} \mathrm{KCl}$, $10 \mathrm{mM}$ DTT, and $0.2 \mu \mathrm{M}$ PMSF. $c$-myc tagged RcTnT was used in our preparation so that the incorporation of exogenously added Tn could be probed using an antibody against the $c-m y c$ epitope. Previous studies have shown that presence of 11 amino acid $c$-myc epitope at the $\mathrm{N}$-terminus of cTnT has no effect on the normal function of the heart [2628]. High salt and urea in the extraction solution containing $c$-myc RcTnT-RcTnI were removed by successive dialysis against the following buffers that contain steadily decreasing salt and urea concentrations: $50 \mathrm{mM}$ Tris- $\mathrm{HCl}(\mathrm{pH} 8.0$ at $4^{\circ} \mathrm{C}$ ), $4 \mathrm{M}$ urea, $0.7 \mathrm{M} \mathrm{KCl}, 1 \mathrm{mM}$ DTT, $4 \mathrm{mM}$ benzamidine$\mathrm{HCl}, 0.4 \mathrm{mM}$ PMSF, and $0.01 \% \mathrm{NaN}_{3}$ followed by $50 \mathrm{mM}$ Tris- $\mathrm{HCl}\left(\mathrm{pH} 8.0\right.$ at $4^{\circ} \mathrm{C}$ ), $2 \mathrm{M}$ urea, $0.5 \mathrm{M} \mathrm{KCl}, 1 \mathrm{mM}$ DTT, $4 \mathrm{mM}$ benzamidine- $\mathrm{HCl}, 0.4 \mathrm{mM}$ PMSF, and $0.01 \%$ $\mathrm{NaN}_{3}$. The extraction solution was then extensively dialyzed against the extraction buffer $\left(50 \mathrm{mM}\right.$ BES ( $\mathrm{pH} 7.0$ at $\left.20^{\circ} \mathrm{C}\right)$, $180 \mathrm{mM} \mathrm{KCl}, 10 \mathrm{mM}$ BDM, $5 \mathrm{mM}$ EGTA, $6.27 \mathrm{mM} \mathrm{MgCl}$, $1.0 \mathrm{mM}$ DTT, $4 \mathrm{mM}$ benzamidine- $\mathrm{HCl}, 0.2 \mathrm{mM}$ PMSF, and $0.01 \% \mathrm{NaN}_{3}$ ). Detergent-skinned papillary muscle fibers were first treated with this $c-m y c$ RcTnT + RcTnI protein sample, followed by RcTnC-WT or RcTnC mutant proteins $(3 \mathrm{mg} / \mathrm{mL}$ ) to complete the reconstitution procedure. In our study, detergent-skinned fiber bundles reconstituted with $c-m y c$ RcTnT + RcTnI + RcTnC-L29Q are referred to as "cTnC $\mathrm{L}_{\mathrm{L} 29 \mathrm{Q}}$ fibers" and those reconstituted with $c-m y c \mathrm{RcTnT}$ $+\mathrm{RcTnI}+\mathrm{RcTnC}-\mathrm{G} 159 \mathrm{D}$ are referred to as $" \mathrm{cTnC}_{\mathrm{G} 159 \mathrm{D}}$ fibers." Fiber bundles reconstituted with $c$-myc RcTnT + $\mathrm{RcTnI}+\mathrm{RcTnC}-\mathrm{WT}$ are referred to as "cTnC $\mathrm{CWT}_{\mathrm{T}}$ fibers" and served as controls in this study.

2.4. SDS-PAGE and Western Blot. We ran 12.5\% SDS PAGE to determine the incorporation of cTnC. First, we used 2\% SDS solution ( $10 \mu \mathrm{L} /$ fiber $)$ to digest the reconstituted fibers for SDS-PAGE, as described previously [29]. SDS-digested fibers were mixed with an equal volume of gel-loading buffer that contained $125 \mathrm{mM}$ Tris- $\mathrm{HCl}(\mathrm{pH} \mathrm{6.8)}, 20 \%$ glycerol, $2 \%$ SDS, $0.01 \%$ bromophenol blue, and $50 \mathrm{mM} \beta$ mercaptoethanol. Digested fibers were run on $12.5 \%$ SDSPAGE to separate the proteins according to their molecular weights $[29,30]$.

For Western blot analysis, proteins from $12.5 \%$ SDSPAGE were transferred onto a PVDF membrane and cTnC was probed using an anti-TnC primary antibody (Clone M5092922, Fitzgerald Industries International, Concord, MA). The resulting protein profiles from the Western blot were used to assess the incorporation of exogenous $\mathrm{cTnC}$ mutants in the reconstituted fibers.

2.5. $p$ Ca Solutions. For $p C a$ titrations, the fiber was bathed in different $\mathrm{pCa}$ solutions ranging from 4.3 to 9.0. The maximal $\mathrm{Ca}^{2+}$-activating solution (pCa 4.3) contained the following (in mM concentrations): $50 \mathrm{BES}, 5 \mathrm{NaN}_{3}, 10$ phosphoenol pyruvate (PEP), 10 EGTA, $10.11 \mathrm{CaCl}_{2}, 6.61 \mathrm{MgCl}_{2}, 5.95$ $\mathrm{Na}_{2} \mathrm{ATP}$, and $31 \mathrm{~K}$-propionate. The relaxing solution (pCa 9.0) contained the following (in $\mathrm{mM}$ concentrations): 50 BES, $5 \mathrm{NaN}_{3}, 10$ PEP, 10 EGTA, $0.024 \mathrm{CaCl}_{2}, 6.87 \mathrm{MgCl}_{2}$,
$5.83 \mathrm{Na}_{2} \mathrm{ATP}$, and $51.14 \mathrm{~K}$-propionate. In addition, pCa solutions contained the following cocktail of protease inhibitors (in $\mu \mathrm{M}$ concentrations): 10 Leupeptin, 1 Pepstatin, 10 PMSF, $20 \mathrm{~A}_{2} \mathrm{P}_{5}$, and 10 Oligomycin. The reagent concentrations of all $\mathrm{pCa}$ solutions were calculated based on a program developed by A. Fabiato and F. Fabiato [31]. The pH of each solution was adjusted to 7.0 using $\mathrm{KOH}$.

2.6. Measurements of Isometric Steady-State Force and ATPase Activity. Isometric steady-state force was measured using methods described previously $[21,22]$. Briefly, detergentskinned muscle fiber was attached between a force transducer and a servo motor using aluminum clips and was submerged in a chamber containing pCa 9.0 solution. The baseline SL of the fiber was adjusted to $2.2 \mu \mathrm{m}$ using laser diffraction technique [32]. The fiber was then activated with a series of pCa solutions starting from pCa 4.3 to 9.0 and the steadystate tension elicited by the fiber in each pCa solution was recorded. These tension values in various $\mathrm{pCa}$ solutions were normalized with its respective value in $\mathrm{pCa}$ 4.3. The normalized tension values were plotted against pCa to construct pCa-tension relationships for each muscle fiber. All measurements from muscle fibers in this study were performed at $20^{\circ} \mathrm{C}$ and at a SL of $2.2 \mu \mathrm{m}$.

$\mathrm{Ca}^{2+}$-activated maximal ATPase activity (pCa 4.3) was measured in reconstituted muscle fibers during isometric steady-state using an assay described previously $[28,33,34]$. In brief, a near UV light was projected through the muscle chamber which was split 50:50 for intensity detection at $340 \mathrm{~nm}$ and $400 \mathrm{~nm}$ wavelengths. Light intensity of the beam at $340 \mathrm{~nm}$ was sensitive to NADH, and thus a change in the UV absorbance at $340 \mathrm{~nm}$ can be directly correlated to the oxidation of NADH (i.e., ATP usage) through enzymatically coupled reactions $[33,34]$. Light intensity of the beam at $400 \mathrm{~nm}$ was insensitive to NADH and, therefore, served as the reference signal. An analog divider and log amplifier produced a signal proportional to the amount of ATP consumed (i.e., amount of NADH oxidized) in the muscle chamber solution. After each recording, the UV absorbance signal of NADH was calibrated by multiple rapid injections of 25 pmol of ADP into the bathing solution, with a motorcontrolled calibration pipette. Tension cost was estimated by dividing the maximal ATPase activity by the maximal tension in each muscle fiber.

2.7. Mechanodynamic Studies. To measure dynamic forcelength relationships, we applied sinusoidal muscle length (ML) changes of constant amplitude $( \pm 0.5 \%$ of $\mathrm{ML})$ to maximally activated muscle fibers $[21,35]$. Two chirps, one with frequencies ranging from 0.1 to $4 \mathrm{~Hz}$ for a time period of $40 \mathrm{~s}$, and the other with frequencies ranging from 1 to $40 \mathrm{~Hz}$ for a time period of $5 \mathrm{~s}$, were administered to emphasize low- and high-frequency force components. The recruitment-distortion (R-D) model was fitted to the overall force response (including both low- and high-frequency components), as described previously [35]. The R-D model predicts a change in muscle force, $\Delta F(t)$, corresponding to 
a change in muscle length, $\Delta \mathrm{ML}(t)$, based on the following equation:

$$
\Delta F(t)=\underbrace{E_{0} \eta(t)}_{\text {recruitment }}+\underbrace{E_{\infty} x(t)}_{\text {distortion }} .
$$

In the equation above, $\eta(t)$ and $x(t)$ are the variables that describe dynamic changes in crossbridge (XB) recruitment and distortion due to changes in ML, respectively. $E_{0}$ and $E_{\infty}$ are stiffness coefficients that are proportional to the number of XB in the states, $\eta(t)$ and $x(t)$, respectively.

The R-D model was fitted to the total force response elicited by the fiber with $\triangle \mathrm{ML}(t)$ as the input to estimate four important model parameters- $E_{0}, b, E_{\infty}, c$ [35]. Previously, we showed that a big advantage of the R-D model is that the total force response (Figure 1(a)) could be uniquely separated into two components: (1) force response due to low-frequency recruitment component $\left(b, E_{0}\right.$; Figure $\left.1(\mathrm{~b})\right)$ and (2) force response due to high-frequency distortion component $\left(c, E_{\infty}\right.$; Figure 1(c)). This feature of the R-D model was successfully used previously to elicit the dynamic features of the respective force components in constantly activated muscle fibers $[21,35]$.

2.8. Crossbridge Model Scheme. In this study, we interpreted our experimental data using a reduced three-state model, as illustrated in Figure 2 [36]. In brief, the model describes thin filament activation using three kinetic processes:

(1) $\mathrm{Ca}^{2+}$ binding to the thin-filament regulatory unit (RU; Tm-Tn);

(2) RU switching between on and off states;

(3) XB cycling between attached and detached states.

To describe these respective processes, the total $\mathrm{XB}$ population is subdivided into two pools: a non-cycling pool $\left(N_{\mathrm{nc}}\right)$ and a cycling pool $\left(N_{c \text {-nfb }}\right.$ and $\left.N_{c \text {-fb }}\right)$. The kinetic processes 1 and 2 are lumped into a single kinetic step that represents RU on/off kinetics. The on/off kinetics of RU are strongly affected by the interactions of thin filament regulatory processes with $\mathrm{Ca}^{2+}$ binding/dissociation kinetics of cTnC. The preferred state of the RU is "off" when $\mathrm{Ca}^{2+}$ is not bound to cTnC, whereas, the preferred state is "on" when $\mathrm{Ca}^{2+}$ is bound to cTnC. When RU are off (i.e., no activator $\mathrm{Ca}^{2+}$ ), the interactions of myosin head with actin are inhibited due to the steric blocking of actin sites by RU. In this scenario, all XB will populate in the non-cycling $N_{\text {nc }}$ state. The binding of $\mathrm{Ca}^{2+}$ to cTnC will switch the RU on by removing their steric blocking effect on actin sites, favoring the entry of XB into the cycling, $N_{c-n f b}$ and $N_{c-\mathrm{fb}}$, states. The rate at which the $\mathrm{XB}$ transition from non-cycling to cycling pool (i.e., RU on kinetics) is represented by $k_{\mathrm{on}}$, whereas, the rate at which XB transition from cycling to noncycling pool (i.e., RU off kinetics) is represented by $k_{\text {off }}$. It is important to note that $k_{\text {on }}$ and $k_{\text {off }}$ are affected by both $\mathrm{Ca}^{2+}$ binding/dissociation kinetics and force-bearing XB. XB within the cycling pool may exist in two distinct states, a nonforce-bearing state, $N_{c \text {-nfb }}$, and a force-bearing state, $N_{c \text {-fb. In }}$ the cycling pool, XB alternate between $N_{c \text {-nfb }}$ and $N_{c \text {-fb }}$ states according to the rate constants, $f$ and $g$.

In the context of this reduced three-state $\mathrm{XB}$ model scheme, the R-D model parameters- $E_{0}, b, E_{\infty}$, and $c-$ specifically represent the following: $E_{\infty}$ is the magnitude of instantaneous length-mediated increase in stiffness due to the rapid distortion of $\mathrm{XB}$ in the $N_{c-\mathrm{fb}}$ state; $E_{0}$ is the magnitude of length-mediated increase in stiffness caused by an increase in the number of $\mathrm{XB}$ in the $N_{c \text {-fb }}$ state; recruitment rate constant, $b$ incorporates various lengthsensing mechanisms including thin filament overlap, XB attachment, and its amplification by cooperativity (indicated by dashed arrow in Figure 2). In other words, the RU on/off rates, $k_{\text {on }}$ and $k_{\text {off }}$, and $\mathrm{XB}$ cycling kinetics defined by rate constants, $f$ and $g$, all coalesce into a single rate constant, $b$. Parameter $c$ governs the distortion dynamics and has a strong dependence on the XB detachment rate, $g$ [35].

2.9. Data Analysis. Data are shown as mean \pm SEM. pCa required to elicit half maximal tension, $p C a_{50}$, and the Hill coefficient, $n_{\mathrm{H}}$, were estimated by fitting the Hill's equation to normalized tension data. Our data included three groups: $c T n C_{W T}, c \operatorname{TnC}_{\mathrm{L} 29 \mathrm{Q}}$, and $\mathrm{cTnC}_{\mathrm{G} 159 \mathrm{D}}$ fibers. Statistical differences between $\mathrm{cTnC}_{\mathrm{WT}}, \mathrm{cTnC}_{\mathrm{L} 29 \mathrm{Q}}$, and $\mathrm{cTnC}_{\mathrm{G} 159 \mathrm{D}}$ fibers were analyzed using one-way ANOVA. Minimal statistical significance was set at $\alpha=0.05$.

\section{Results}

3.1. Incorporation of Recombinant Mutant cTnC Proteins into Myofibers. Figure 3 shows the cTnC protein profiles from various reconstituted muscle fiber groups. The Western blot against anti-cTnC primary antibody confirmed the absence of native cTnC in the cTnT-cTnI treated fiber bundles, clearly demonstrating a near-complete removal of endogenous Tn units using our exchange procedure (Figure 3; lane 3). Furthermore, the Western blot confirmed that the recombinant $\mathrm{cTnC}_{\mathrm{L} 29 \mathrm{Q}}$ (lane 4) and $\mathrm{cTnC}_{\mathrm{G} 159 \mathrm{D}}$ (lane 5) mutants were incorporated properly in the reconstituted fiber bundles.

3.2. Effect of $c T n C_{L 29 Q}$ and $c T n C_{G 159 D}$ on $\mathrm{Ca}^{2+}$-Activated Maximal Tension, Maximal ATPase Activity, and the Magnitude of Myofiber Dynamic Stiffness. We first assessed the effects of $\mathrm{cTnC}_{\mathrm{L} 29 \mathrm{Q}}$ and $\mathrm{cTnC}_{\mathrm{G} 159 \mathrm{D}}$ mutants on $\mathrm{Ca}^{2+}$ activated maximal tension and maximal ATPase activity. To determine the maximal tension, reconstituted fibers were bathed in pCa 4.3 solution until they reached a steadystate isometric force. The isometric steady-state force was then converted to tension by expressing it as force per cross-sectional area. The $\mathrm{Ca}^{2+}$-activated maximal tension values (in $\mathrm{mN} \cdot \mathrm{mm}^{-2}$ ) in $\mathrm{cTnC}_{\mathrm{WT}}, \mathrm{cTnC}_{\mathrm{L} 29 \mathrm{Q}}$, and $\mathrm{cTnC}_{\mathrm{G} 159 \mathrm{D}}$ fibers were $57 \pm 2(n=15), 56 \pm 2(n=10)$, and $53 \pm 2(n=14)$, respectively. These data demonstrate that the $\mathrm{Ca}^{2+}$-activated maximal tension values in $\mathrm{cTnC}_{\mathrm{L}_{29} \mathrm{Q}^{-}}$ and $\mathrm{cTnC}_{\mathrm{G} 159 \mathrm{D}}$-reconstituted fibers were similar to that of $\mathrm{cTnC}_{\mathrm{WT}}$-reconstituted fibers. Thus, both $\mathrm{cTnC}_{\mathrm{L} 29 \mathrm{Q}}$ and $\mathrm{cTnC}_{\mathrm{G} 159 \mathrm{D}}$ had no impact on $\mathrm{Ca}^{2+}$-activated maximal tension. Our observations in maximal tension agree well with many previous in vitro studies of L29Q $[10,14,15]$ and 


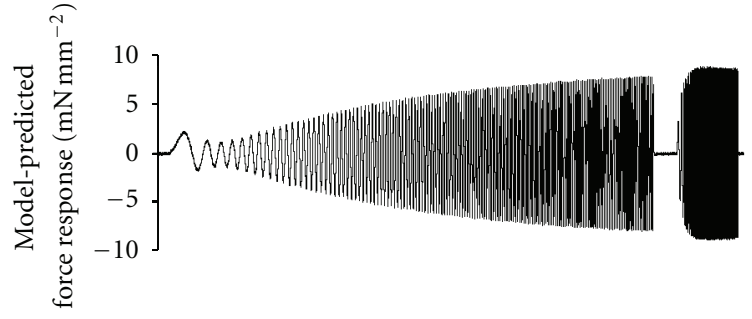

(a)

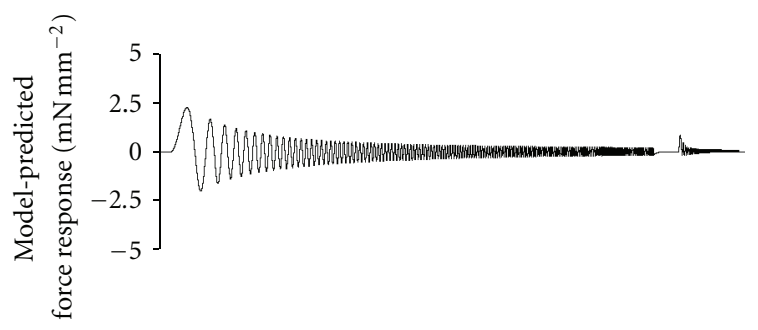

(b)

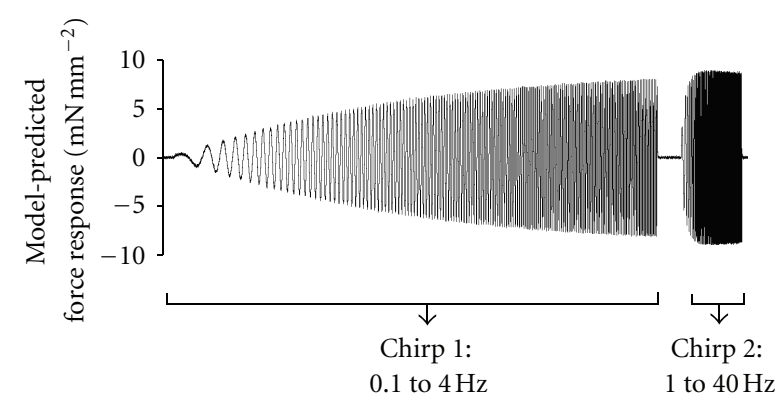

(c)

FIGURE 1: Representative model-predicted force response to chirp-length perturbation for a control-rat fiber (i.e., cTnC $\mathrm{CWT}_{W \mathrm{~T}}$ fiber). (a) Total force response that includes force components due to low-frequency XB recruitment component, $E_{0}{ }^{*} \eta(t)$, and high-frequency XB distortion component, $E_{\infty}{ }^{*} x(t)$. (b) Force response due to low-frequency XB recruitment component, $E_{0}{ }^{*} \eta(t)$. (c) Force response due to high-frequency $\mathrm{XB}$ distortion component, $E_{\infty}{ }^{*} x(t) . E_{0}$ and $E_{\infty}$ represent stiffness magnitudes that scale the contributions of recruitment and distortion components to total stiffness. $\eta(t)$ and $x(t)$ are variables that describe the dynamic changes in the XB recruitment and distortion components due to changes in muscle length. Two chirps were administered to emphasize low- and high-frequency components of the force response: from 0.1 to $4 \mathrm{~Hz}$ over $40 \mathrm{~s}$ in chirp 1 and from 1 to $40 \mathrm{~Hz}$ over $5 \mathrm{~s}$ in chirp 2 .

G159D $[14,16,19,20]$ mutations, confirming that both these mutants did not affect the maximal tension. Thus, our tension data further substantiated our conclusion from the Western blot (Figure 3) that the reconstitution of $\mathrm{cTnC}_{\mathrm{L} 29 \mathrm{Q}}$ and $\mathrm{cTnC}_{\mathrm{G159D}}$ mutants into detergent-skinned fibers was normal.

We also measured the maximal ATPase activity in reconstituted muscle fibers using a procedure described in Methods. The $\mathrm{Ca}^{2+}$-activated maximal ATPase values (in pmol . $\left.\mathrm{mm}^{-3} \cdot \mathrm{s}^{-1}\right)$ in $\mathrm{cTnC} \mathrm{CT}_{\mathrm{T}}, \mathrm{cTnC}_{\mathrm{L} 29 \mathrm{Q}}$, and $\mathrm{cTnC}_{\mathrm{G} 159 \mathrm{D}}$ fibers were $193 \pm 9(n=9), 187 \pm 8(n=8)$, and $219 \pm 13(n=9)$, respectively. Although the maximal ATPase activity was not significantly different between various groups, $\mathrm{cTnC}_{\mathrm{G} 159 \mathrm{D}}$ fibers demonstrated an increasing trend in the maximal ATPase consumption (by $13.4 \%$ ) when compared to that of cTnCwT fibers.

We also estimated the magnitudes of $\mathrm{XB}$ distortion dynamics $\left(E_{\infty}\right)$ and recruitment dynamics $\left(E_{0}\right)$ in $\mathrm{cTnC}_{\mathrm{WT}^{-}}, \mathrm{cTnC}_{\mathrm{L}_{29 \mathrm{Q}^{-}}}$, and $\mathrm{cTnC}_{\mathrm{G}_{159 \mathrm{D}}}$-reconstituted fibers. In previous studies, we have demonstrated that $E_{\infty}$ is a measure of the number of strongly-bound $\mathrm{XB}$ and $E_{0}$ is a measure of the number of newly-recruited $\mathrm{XB}$ due to an increase in muscle length [22]. Both $E_{0}$ and $E_{\infty}$ estimates in $\mathrm{cTnC}_{\mathrm{L} 29 \mathrm{Q}^{-}}$and $\mathrm{cTnC}_{\mathrm{G} 159 \mathrm{D}}$-reconstituted fibers were not significantly different from those of $\mathrm{cTnC}_{\mathrm{WT}}$-reconstituted fibers. $E_{0}$ estimates (in $\mathrm{mN} \mathrm{mm}^{-3}$ ) in cTnC $\mathrm{WT}_{\mathrm{WT}}, \mathrm{cTnC}_{\mathrm{L} 29 \mathrm{Q}}$, and $c \operatorname{TnC}_{\mathrm{G} 159 \mathrm{D}}$ fibers were $173 \pm 10(n=15), 151 \pm 10(n=$ $10)$, and $148 \pm 11(n=14)$, respectively. The corresponding
$E_{\infty}$ estimates (in $\mathrm{mN} \mathrm{mm}^{-3}$ ) were $786 \pm 32(n=15), 750 \pm 47$ $(n=10)$, and $788 \pm 43(n=14)$, respectively. Thus, both ${ } T_{n n} C_{\mathrm{L} 29}$ and $\mathrm{cTnC}_{\mathrm{G} 159 \mathrm{D}}$ had no effect on the number of strongly-bound $\mathrm{XB}$ and the number of newly-recruited $\mathrm{XB}$ due to a change in muscle length. Collectively, our results from the Western blot, $\mathrm{Ca}^{2+}$-activated maximal tension, and the magnitude of $\mathrm{XB}$ distortion dynamics indicate that both cTnC mutants incorporated properly into the myofibers and that they had no effect on either the number of stronglybound $\mathrm{XB}$ or maximal tension.

3.3. Effect of $c T n C_{L 29 Q}$ and $c T n C_{G 159 D}$ on Myofilament $\mathrm{Ca}^{2+}$ Sensitivity and Cooperativity. To determine if $\mathrm{CTnC}_{\mathrm{L} 29 \mathrm{Q}}$ and $c_{\mathrm{TnC}} \mathrm{G}_{\mathrm{G} 9 \mathrm{D}}$ mutants altered myofilament $\mathrm{Ca}^{2+}$ sensitivity $\left(p C a_{50}\right)$ and cooperativity $\left(n_{\mathrm{H}}\right)$, we fitted the Hill's equation to normalized tension data obtained at different $\mathrm{pCa}$. Figure 4 illustrates a comparison of pCa-tension relationships between $c \mathrm{TnC}_{\mathrm{WT}}$, $\mathrm{cTnC}_{\mathrm{L} 29 \mathrm{Q}}$, and $\mathrm{cTnC}_{\mathrm{G} 159 \mathrm{D}}$ fibers. When compared to $\mathrm{cTnC}_{\mathrm{WT}}$ fibers, $\mathrm{cTnC}_{\mathrm{L} 29 \mathrm{Q}}$ fibers showed a small but significant decrease in $p C a_{50}$, as indicated by a rightward shift in the pCa-tension relationships $(P<$ 0.001 ; Figures 4 and $5(\mathrm{a}))$. However, estimates of $p C a_{50}$ in $\mathrm{cTnC}_{\mathrm{G159D}}$ fibers were not different from those of $\mathrm{cTnC}_{\mathrm{WT}} \mathrm{T}^{-}$ reconstituted fibers (Figures 4 and 5(a)), suggesting that $\mathrm{cTnC}_{\mathrm{G} 159 \mathrm{D}}$ did not affect myofilament $\mathrm{Ca}^{2+}$ sensitivity. These data demonstrate that $\mathrm{cTnC}_{\mathrm{L} 29 \mathrm{Q}}$ and $\mathrm{cTnC}_{\mathrm{G} 159 \mathrm{D}}$ behave differently, with respect to their effect on myofilament $\mathrm{Ca}^{2+}$ sensitivity. $n_{\mathrm{H}}$ was unaltered in fibers reconstituted 


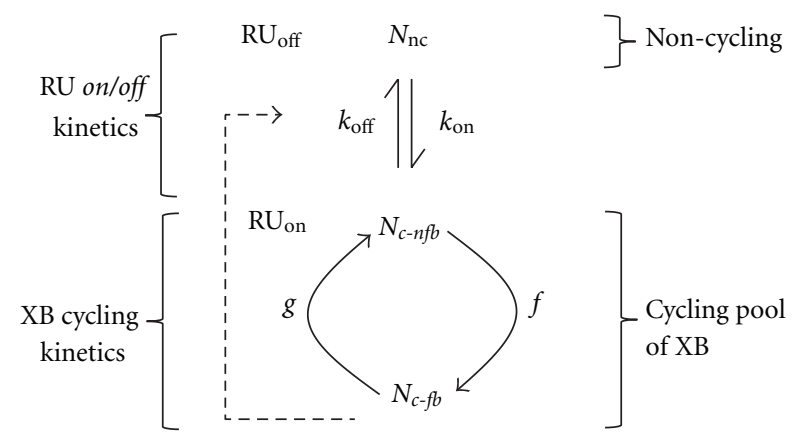

FIgUre 2: Reduced three-state crossbridge (XB) model scheme depicting regulatory unit (RU; Tm-Tn) kinetics and XB cycling kinetics. This scheme is adapted from Campbell [36]. $k_{\text {on }}$ and $k_{\text {off }}$, represent the RU on/off rates and are functions of $\mathrm{Ca}^{2+}$ bound to cTnC. Once turned on by the binding $\mathrm{Ca}^{2+}$, RU permits the transition of $\mathrm{XB}$ from the non-cycling $\left(N_{\mathrm{nc}}\right)$ pool to the cycling pool of XB. The cycling pool of XB includes two states of XB: cycling non-force-bearing $\left(N_{c-n f b}\right)$ and cycling force-bearing $\left(N_{c-f b}\right)$. The transition between non-cycling and cycling pools is mainly regulated by $k_{\text {on }} / k_{\text {off }}$ kinetics of RU. The influence of the forcebearing XB on the RU on/off kinetics is represented by the feedback arrow (dashed line). $f$ and $g$ represent the rate constants governing forward transition, $N_{c-\mathrm{nfb}} \rightarrow N_{c-\mathrm{fb}}$, and backward transition, $N_{c-\mathrm{fb}} \rightarrow$ $N_{c-\text { nfb }}$, respectively.

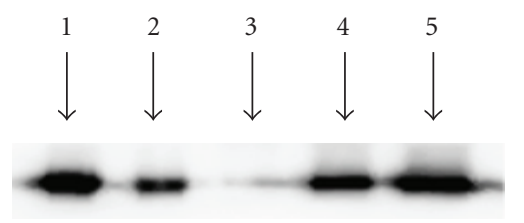

Figure 3: Western blot analysis of detergent-skinned rat cardiac muscle fibers reconstituted with $\mathrm{cTnC}_{\mathrm{WT}}, \mathrm{cTnC}_{\mathrm{L} 29 \mathrm{Q}}$ or $\mathrm{cTnC}_{\mathrm{G} 159 \mathrm{D}}$. Muscle protein samples were separated on $12.5 \%$ SDS-PAGE. Proteins from gel were transferred to a PVDF membrane and RcTnC was probed with an anti-TnC primary antibody (Fitzgerald M5092922). Protein profiles in lanes 1-5 represent the following: lane 1, purified RcTnC; lane 2, fibers reconstituted with $c$-myc $\mathrm{RcTnT}+\mathrm{RcTnI}+\mathrm{RcTnC}-\mathrm{WT}$; lane 3, fibers reconstituted with $c$ $m y c$ RcTnT + RcTnI; lane 4, fibers reconstituted with $c$-myc RcTnT $+\mathrm{RcTnI}+\mathrm{RcTnC}$-L29Q; lane 5, fibers reconstituted with $c$-myc RcTnT + RcTnI + RcTnC-G159D.

with either $\mathrm{cTnC}_{\mathrm{L} 29 \mathrm{Q}}$ or $c \mathrm{Cnn}_{\mathrm{G} 159 \mathrm{D}}$, suggesting that the myofilament cooperativity was unaffected by both cTnC mutants (Figure 5(b)).

3.4. Effect of $c T n C_{L 29 Q}$ and $c T n C_{G 159 D}$ on $X B$ Detachment Kinetics. Myofilament $\mathrm{Ca}^{2+}$ sensitivity may also be affected by changes in XB detachment kinetics, $g$. For example, an increase in $g$ may also decrease myofilament $\mathrm{Ca}^{2+}$ sensitivity. To examine whether $\mathrm{cTnC}$ mutations affected the XB detachment kinetics, we estimated length-mediated $\mathrm{XB}$ distortion dynamic, $c$, and tension cost, TC, in detergent-skinned muscle bundles reconstituted with $\mathrm{cTnC}_{\mathrm{WT}}, \mathrm{cTnC}_{\mathrm{L} 29 \mathrm{Q}}$, or $\mathrm{cTnC}_{\mathrm{G159D}}$ mutants. Previously, we have shown that TC is

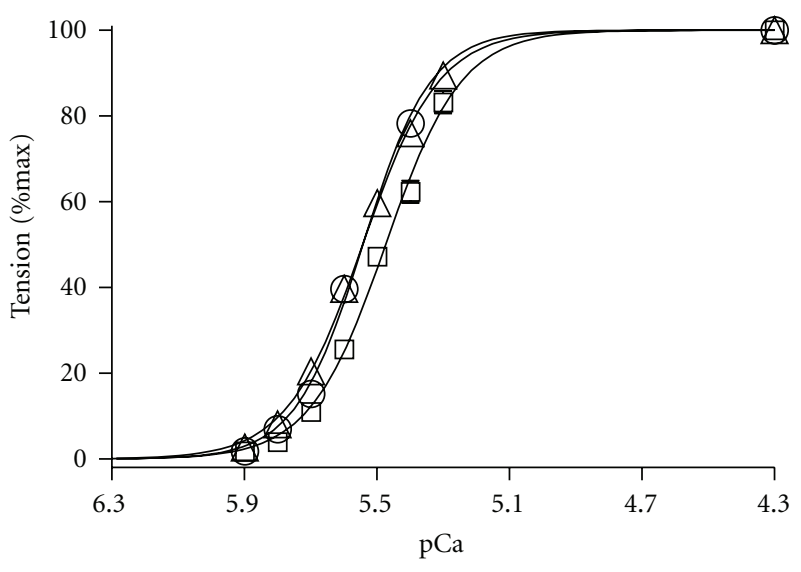

FIGURE 4: Comparison of normalized pCa-tension relationships in detergent-skinned fibers reconstituted with $\mathrm{cTnC}_{\mathrm{WT}}, \mathrm{cTnC}_{\mathrm{L} 29 \mathrm{Q}}$, or $\mathrm{cTnC}_{\mathrm{G} 159 \mathrm{D}}$. Isometric steady-state tensions elicited by each fiber in various $\mathrm{pCa}$ solutions were normalized with its respective value in $\mathrm{pCa} 4.3$ solution. The normalized tensions were plotted against pCa to construct the pCa-tension relationship. The Hill equation was fitted to the normalized $\mathrm{pCa}$-tension relationships to estimate myofilament $\mathrm{Ca}^{2+}$ sensitivity $\left(\mathrm{pCa}_{50}\right)$ and the Hill coefficient $\left(n_{\mathrm{H}}\right), p C a_{50}$ and $n_{\mathrm{H}}$ values are shown in Figures 5(a) and 5(b), respectively. The curves presented here are Hill fits to pCa-tension relationships in $\mathrm{cTnC}_{\mathrm{WT}}(\bigcirc), \mathrm{cTnC}_{\mathrm{L} 29 \mathrm{Q}}(\square)$, and $\mathrm{cTnC}_{\mathrm{G} 159 \mathrm{D}}(\triangle)$ fibers, respectively. Values are expressed as mean \pm SEM. Number of fibers tested in each group is as follows: $\mathrm{cTnC}_{\mathrm{WT}}, n=15 ; \mathrm{cTnC}_{\mathrm{L} 29 \mathrm{Q}}, n=10 ; \mathrm{cTnC}_{\mathrm{G} 159 \mathrm{D}}, n=14$.

strongly correlated to $c$ and that both have a strong dependence on the XB detachment rate, $g$ [35]. Thus, changes in $c$ and TC may convey important effects of $\mathrm{cTnC}_{\mathrm{L} 29 \mathrm{Q}}$ and $\mathrm{cTnC}_{\mathrm{G} 159 \mathrm{D}}$ on XB detachment kinetics. Our estimates of $c$ and $\mathrm{TC}$ in $\mathrm{CTnC}_{\mathrm{L} 29 \mathrm{Q}}$ fibers were not significantly different from those of $\mathrm{cTnC}_{\mathrm{WT}}$ fibers (Figures 6(a) and 6(b), resp.). However, estimates of $c$ and $\mathrm{TC}$ in $\mathrm{cTnC}_{\mathrm{G} 159 \mathrm{D}}$ fibers were significantly higher by $15 \%(P<0.01$; Figure $6(\mathrm{a}))$ and $26 \%(P<0.01$; Figure 6(b)), respectively, suggesting that the $\mathrm{CTnC}_{\mathrm{G} 159 \mathrm{D}}$ mutant increased $\mathrm{XB}$ detachment rate. It is important to note that although the maximal tension and maximal ATPase activity of $\mathrm{cTnC}_{\mathrm{G} 159 \mathrm{D}}$ fibers were not significantly different from those of $\mathrm{cTnC}_{\mathrm{WT}}$ fibers, the TC was significantly higher. The reason for this is that the maximal tension is slightly lower (by 7\%) and the maximal ATPase is slightly higher (by $13.4 \%$ ), making the $\mathrm{TC}$ significantly higher in $\mathrm{cTnC}_{\mathrm{G} 159 \mathrm{D}}$ fibers when compared to that of $\mathrm{cTnC}_{\mathrm{WT}}$ fibers.

3.5. Effect of $c T n C_{L 29 Q}$ and $c T n C_{G 159 D}$ on the Rate of $X B$ Recruitment Dynamics. cTnC mutant-induced effect on myofilament $\mathrm{Ca}^{2+}$ sensitivity may indicate an effect on RU on/off kinetics. Because changes in RU on/off kinetics have an impact on the rate of $\mathrm{XB}$ recruitment dynamics, we measured the rate constant of $\mathrm{XB}$ recruitment dynamics, $b$, using dynamic muscle fiber stiffness measurements in

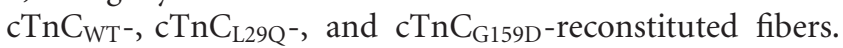
As illustrated in Figure 7, our observations show that $b$ speeds by $26.5 \%(P<0.01)$ in $\mathrm{CTnC}_{\mathrm{L} 29 \mathrm{Q}}$ and by 


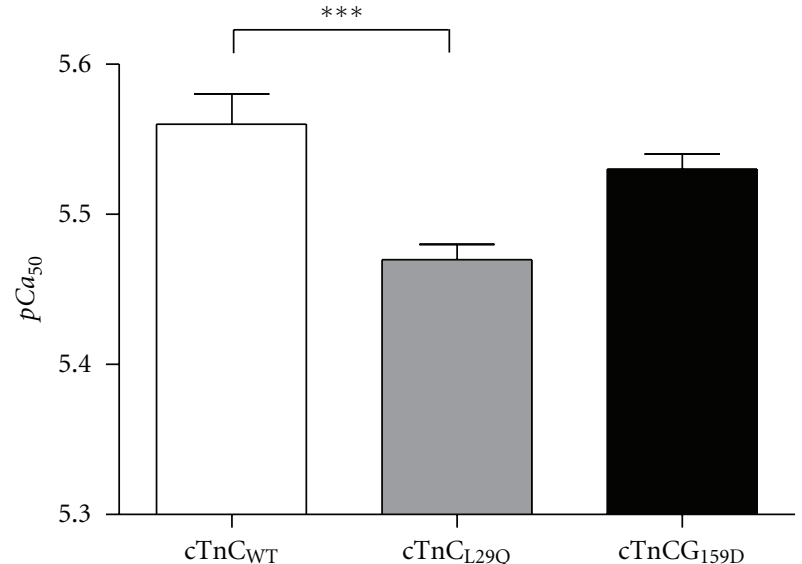

(a)

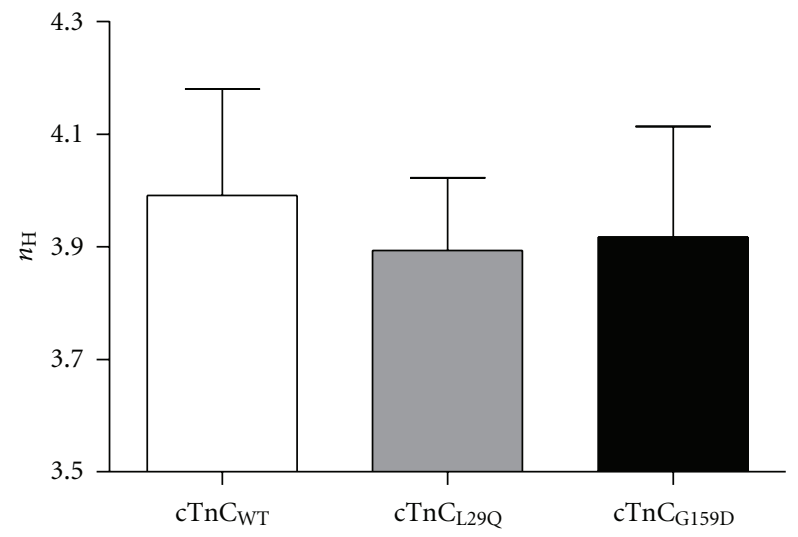

(b)

FIGURE 5: Comparison of myofilament $\mathrm{Ca}^{2+}$ sensitivity $\left(p C a_{50}\right)$ and cooperativity $\left(n_{\mathrm{H}}\right)$ of pCa-tension relationships in detergent-skinned fibers reconstituted with $\mathrm{cTnC}_{\mathrm{WT}}, \mathrm{ccTnC}_{\mathrm{L} 29 \mathrm{Q}}$, or $\mathrm{cTnC}_{\mathrm{G} 159 \mathrm{D}}$. (a) Effects of RcTnC mutants on $p C a_{50}$ (b) Effects of RcTnC mutants on $n_{\mathrm{H}}$. The Hill equation was fitted to the normalized pCa-tension relationships to estimate $p C a_{50}$ and $n_{\mathrm{H}}$. One-way ANOVA was used to compare $p C a_{50}$ and $n_{\mathrm{H}}$ estimates in $\mathrm{cTnC}_{\mathrm{L} 29 \mathrm{Q}}$ and $\mathrm{cTnC}_{\mathrm{G} 159 \mathrm{D}}$ fibers with the data from cTnC $\mathrm{WT}_{\mathrm{T}}$ fibers as controls. Values are expressed as mean $\pm \mathrm{SEM}$. Number of fibers tested in each group is as follows: $\mathrm{cTnC}_{\mathrm{WT}}, n=15 ; \mathrm{cTnC}_{\mathrm{L} 29 \mathrm{Q}}, n=10 ; \mathrm{cTnC}_{\mathrm{G} 159 \mathrm{D}}, n=14$. Minimal statistical significance was set at $\alpha=0.05$. ${ }^{* * *} P<0.001$.

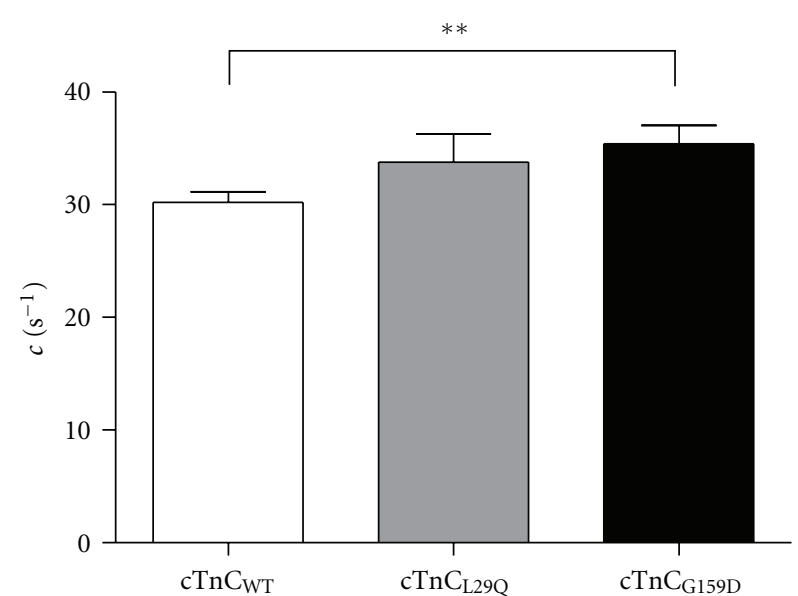

(a)

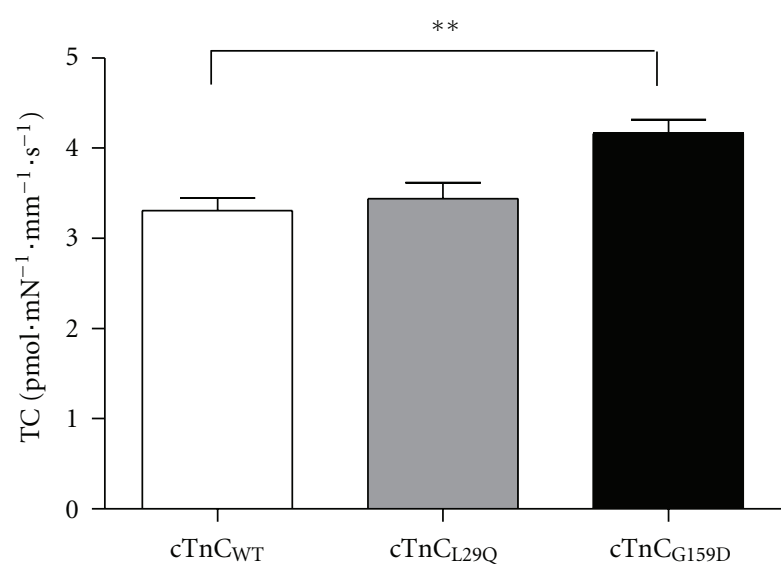

(b)

FIgure 6: Comparison of $\mathrm{XB}$ distortion rate constant, $c$, and tension cost, $\mathrm{TC}$, in detergent-skinned fibers reconstituted with $c \operatorname{TnC}_{\mathrm{WT}}, \mathrm{cTnC}_{\mathrm{L} 29 \mathrm{Q}}$, or $\mathrm{cTnC}_{\mathrm{G} 159 \mathrm{D}}$. (a) Effects of RcTnC mutants on $c$. $c$ was estimated by fitting the R-D model to the force responses from muscle fibers to chirp-length perturbations [35]. (b) Effects of RcTnC mutants on TC. TC was estimated by dividing the maximal ATPase activity with the maximal tension elicited by the muscle fiber. One-way ANOVA was used to compare estimates of $c$ and TC in $\mathrm{cTnC}_{\mathrm{L} 29 \mathrm{Q}}$ and $\mathrm{cTnC}_{\mathrm{G} 159 \mathrm{D}}$ fibers with the data from $\mathrm{cTnC}_{\mathrm{WT}}$ fibers as controls. Values are reported as mean \pm SEM. Number of fibers tested in each group is as follows: $\mathrm{cTnC}_{\mathrm{WT}}, n=15 ; \mathrm{cTnC}_{\mathrm{L} 29 \mathrm{Q}}, n=10 ; \mathrm{cTnC}_{\mathrm{G} 159 \mathrm{D}}, n=14$. Minimal statistical significance was set at $\alpha=0.05$, $* * P<0.01$.

$25.3 \%(P<0.05)$ in $\mathrm{cTnC}_{\mathrm{G} 159 \mathrm{D}}$ fibers. Therefore, our data suggest that both $\mathrm{cTnC}_{\mathrm{L} 29 \mathrm{Q}}$ and $\mathrm{cTnC}_{\mathrm{G} 159 \mathrm{D}}$ mutants affect thin filament processes that mediate the length-dependent effects on the rate of XB recruitment dynamics.

\section{Discussion}

Experiments presented here provide new evidence for the mechanism by which $\mathrm{TnC}$ mutations bring about global change in myofilament mechanodynamics. To our knowledge, this is the first study that addresses important unresolved questions. (1) What is the effect of TnC mutations on $\mathrm{XB}$ recruitment and distortion dynamics? (2) How does the interplay between $\mathrm{Ca}^{2+}$ binding kinetics and $\mathrm{XB}$ cycling kinetics produce a global change in myofilament mechanodynamics? Global myofilament kinetics is governed by interactions between $\mathrm{Ca}^{2+}$ binding kinetics and $\mathrm{XB}$ cycling kinetics $[7,8,36]$. We have tested this interplay 


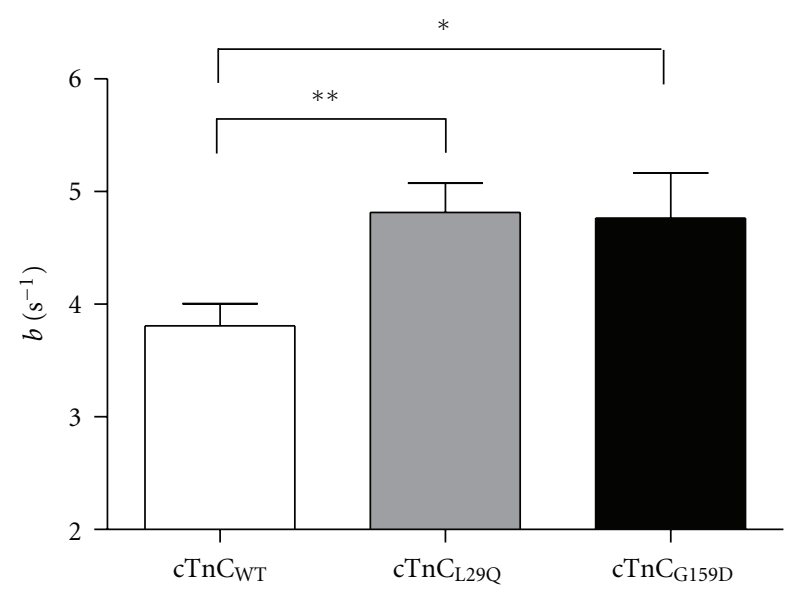

Figure 7: Comparison of $\mathrm{XB}$ recruitment dynamic, $b$, in detergent-skinned fibers reconstituted with $\mathrm{cTnC}_{\mathrm{WT}}, \mathrm{cTnC}_{\mathrm{L} 29 \mathrm{Q}}$, or $\mathrm{cTnC}_{\mathrm{G} 159 \mathrm{D}}$. $b$ was estimated by fitting the R-D model to the force responses from muscle fibers to chirp-length perturbations [35]. One-way ANOVA was used to compare estimates of $b$ in $\mathrm{cTnC}_{\mathrm{L} 29 \mathrm{Q}}$ and $\mathrm{cTnC}_{\mathrm{G} 159 \mathrm{D}}$ fibers with the data from $\mathrm{cTnC}_{\mathrm{WT}}$ fibers as controls. Values are expressed as mean \pm SEM. Number of fibers tested in each group is as follows: $\mathrm{cTnC}_{\mathrm{WT}}, n=15$; $\mathrm{cTnC}_{\mathrm{L} 29 \mathrm{Q}}, n=10$; $\mathrm{cTnC}_{\mathrm{G} 159 \mathrm{D}}, n=14$. Minimal statistical significance was set at $\alpha=$ 0.05. ${ }^{*} P<0.05$; ${ }^{* *} P<0.01$

effect by measuring pCa-tension relationship and myofiber dynamic stiffness in rat cardiac muscle fibers reconstituted with L29Q and G159D cTnC mutants. New data from our study provides a mechanistic basis for the functional effects observed in humans containing L29Q and G159D mutations in $\mathrm{cTnC}$.

Our finding that the L29Q mutation elicited a small but significant decrease in myofilament $\mathrm{Ca}^{2+}$ sensitivity, $p C a_{50}$ $(\sim 0.09$ units; Figure 5(a)), is consistent with the report from a previous study [11]. Furthermore, our observation that $p C a_{50}$ remained unaltered by the G159D mutation is also in agreement with previous studies which employed reconstituted assays $[19,20]$. However, there are significant discrepancies between our observations and other studies, which reported contrasting findings on $p C a_{50}$ for either L29Q [10, 14, 15] or G159D mutation [14, 16-18]. Some of these discrepancies between our study and others [10, 14-18] may be attributed to many experimental variants, including but not limited to the type of proteins used (i.e., homologous or heterologous), reconstitution techniques employed, phosphorylation status of cTnI in the reconstituted system, species-specific differences (mouse, rat, pig, rabbit, human), and so forth.

Our study showed a decrease in myofilament $\mathrm{Ca}^{2+}$ sensitivity with the L29Q mutation, while a previous study showed no effect [15]. On the other hand, our finding that G159D mutation had no effect on $\mathrm{Ca}^{2+}$ sensitivity is in contrast with two previous studies which showed a decrease $[17,18]$. Such discrepancies between our study and the aforementioned studies may be likely due to the use of heterologous proteins. For example, while Neulen et al. [15] reconstituted human cardiac Tn subunits into mouse cardiac myofilaments, Mirza et al. [17] and Robinson et al. [18] used rabbit skeletal (F-actin and myosin) and human cardiac ( TnC/I/T and Tm) muscle proteins in their in vitro ATPase assays. The use of such a heterologous reconstituted system to understand the functional effect of a single site mutation in $\mathrm{cTnC}$ makes it difficult to ascribe the findings directly to the specific substitution introduced. Our study avoids this issue through reconstitution of rat papillary muscle fibers with homologous recombinant rat cardiac Tn subunits.

The method used for reconstituting the recombinant proteins into the thin filament may also play a role in such discrepancies. For example, two previous investigations used CDTA treatment to selectively extract the endogenous cTnC subunits in their experimental preparations and to reconstitute them with recombinant cTnC mutants $[14,15]$. In this regard, our study differs in that we removed all endogenous cardiac Tn subunits from rat papillary muscle fibers and reconstituted them with recombinant rat cardiac Tn subunits. Furthermore, because Dweck et al. [14] and Neulen et al. [15] confined the extraction and reconstitution to $\mathrm{CTnC}$ in their studies, it may be possible that phosphorylation of endogenous cTnI in their experimental preparations might be different from our preparations (reconstituted with nonphosphorylated cTnI). In addition, functional effects can also be attributed to the use of lower rodents (rats in our study) versus larger animals (pigs in the study by Dweck et al. [14] and humans in the study by Dyer et al. [16]). These possible factors may likely explain the discrepancies observed between our study and the aforementioned studies of L29Q and G159D mutations.

The first question that needs to be addressed in our study is, "how does the L29Q mutation brings about a small change in $\mathrm{Ca}^{2+}$ sensitivity?" The L29Q mutation may affect myofilament $\mathrm{Ca}^{2+}$ sensitivity via either a direct effect on $\mathrm{Ca}^{2+}$ binding to site-II of TnC or an indirect allosteric effect on the overall configuration of the regulatory unit (RU; Tn-Tm). Evidence pertaining to these claims comes from previous $\mathrm{Ca}^{2+}$ binding affinity studies of L29Q mutation, which suggest a possible L29Q-induced destabilization of helix A in cTnC. This effect of L29Q on helix A may affect the $\mathrm{Ca}^{2+}$ binding properties at site II [10] and/or the interaction between cTnC and cTnI [11]. Regardless of the way the L29Q mutation affects myofilament $\mathrm{Ca}^{2+}$ sensitivity, we expect that the kinetics of on/off transition of the RU will be affected. Based on the XB model scheme shown in Figure 2, we think that a decrease in $\mathrm{Ca}^{2+}$ sensitivity may be linked to RU on/off kinetics, $k_{\text {on }}$ and $k_{\text {off }}$. Because force-bearing XB have an effect on on/off kinetics of RU, we first established that the infinite frequency stiffness $\left(E_{\infty}\right)$ was not altered in L29Q-reconstituted fibers. Previously, we have demonstrated that $E_{\infty}$ and $\mathrm{Ca}^{2+}$-activated maximal tension $\left(T_{\max }\right)$ are both measures of the number of parallel force-bearing XB [35]. Our observations that $E_{\infty}$ and $T_{\max }$ were unaltered suggested that the L29Q mutation did not affect the number of force-bearing XB. Another mechanism that may decrease myofilament $\mathrm{Ca}^{2+}$ sensitivity is through an augmenting effect on $\mathrm{XB}$ detachment rate, $g$. Our observation that both the rate constant for $\mathrm{XB}$ distortion dynamics $(c)$ and tension cost (TC) were unaltered suggested that the L29Q mutation 
did not affect $g$ (Figures 6(a) and 6(b), resp.). Our finding is consistent with a previous study which showed that the velocity of unloaded shortening was unaltered by the L29Q mutation [15]. Collectively, these observations suggest that the L29Q mutation may affect the equilibrium between the non-cycling and cycling XB pools by affecting the $k_{\text {on }} / k_{\text {off }}$ of RU (Figure 2).

An impact on $k_{\text {on }} / k_{\text {off }}$ of RU will affect the dynamics of $\mathrm{XB}$ recruitment $[22,35]$. To determine the effects on $\mathrm{XB}$ dynamics, we assessed the length-mediated effects on $\mathrm{XB}$ recruitment rate, $b$, in $\mathrm{L} 29 \mathrm{Q}$-reconstituted fibers. $b$ increased by $26.5 \%$ in L29Q-reconstituted fibers. It is important to note that dynamics of $\mathrm{XB}$ recruitment are affected by various length-sensing mechanisms, including changes in the thin filament overlap and XB cycling kinetics [35]. In addition, $\mathrm{XB}$ themselves affect the balance between RU on/off states via cooperative mechanisms (dashed line with feedback arrow in Figure 2). Therefore, $b$ is a function of the RU on/off rates, $k_{\text {on }}$ and $k_{\text {off }}$, as well as the rate parameters that define $\mathrm{XB}$ cycling kinetics, $f$ and $g$ [35]. Because $g$ was unaffected by the L29Q mutation, an increase in $b$ may be associated with an increase in any of the following rate constants$k_{\text {off }}, k_{\text {on }}$, and $f$ (Figure 2). However, an increase in $k_{\text {on }}$ or $f$ is unlikely because such effects would increase the number of force-bearing $\mathrm{XB}$ and the $T_{\max }$, effects that are not observed in L29Q-reconstituted fibers. Thus, we predict that an increase in $b$ in L29Q-reconstituted fibers is probably due to an increase in the RU off rate, $k_{\text {off }}$. Because $k_{\text {on }}$ and $k_{\text {off }}$ are functions of $\mathrm{Ca}^{2+}$ bound to $\mathrm{RU}$, an increase in $k_{\text {off }}$ is consistent with a decrease in $p C a_{50}$ observed in L29Qreconstituted fibers. A higher $k_{\text {off }}$ stabilizes $\mathrm{XB}$ in the $N_{\mathrm{nc}}$ state, thereby reducing their transitioning into the cycling pool, $N_{c \text {-nfb }}$ (Figure 2). Because the population of XB in the cycling pool depends on the net effect of $k_{\text {on }}$ and $k_{\text {off }}$, an increase in $k_{\text {off }}$ acts to reduce $\mathrm{XB}$ recruitment, resulting in a lower tension in $\mathrm{L} 29 \mathrm{Q}$ fibers at submaximal $\mathrm{Ca}^{2+}$ activations. However, at maximal $\mathrm{Ca}^{2+}$ activation, all the troponin units have near-maximal $\mathrm{Ca}^{2+}$ bound to $\mathrm{TnC}$, thereby increasing the probability of more force-bearing XB (i.e., $f$ ). Thus, the net effect of $k_{\text {off }}$ on $f$ will be minimal at maximal $\mathrm{Ca}^{2+}$ activation, resulting in no effect on the number of forcebearing $\mathrm{XB}$ and the maximal force.

The G159D mutation had no effect on $p C a_{50}, T_{\max }$, and $E_{\infty}$. Thus, its effect on the heart must come from another source of primary myofilament defect that remains poorly understood. Our measurements in G159D-reconstituted fibers showed that both the rate constant for $\mathrm{XB}$ distortion dynamic, $c$, and TC were significantly higher in G159D fibers (Figures 6(a) and 6(b)). Because both $c$ and TC have a strong dependence on $g[21,35]$, higher $c$ and TC are indicative of an increase in the rate of XB detachment in G159D fibers. In addition, G159D fibers also demonstrated a significant increase in the rate constant for XB recruitment dynamic, $b$ (Figure 7). As discussed before, an increase in $b$ may be associated with an increase in any of the rate constants$k_{\text {on }}, k_{\text {off }}, f$, and $g$. However, an unaltered $p C a_{50}$ supports the notion that the RU on/off rates, $k_{\text {on }}$ and $k_{\text {off }}$, are not affected by the G159D mutation. Therefore, an increase in $b$ may be attributed to an increase in $g$ alone or an increase in both $f$ and $g$. An increase in $g$ alone is unlikely because such an effect would reduce the number of force-bearing $\mathrm{XB}$, which would decrease both $T_{\max }$ and myofilament $\mathrm{Ca}^{2+}$ sensitivity. Therefore, a plausible interpretation is that the G159D mutant may increase both $f$ and $g$, causing XB to cycle faster. Although conjectural, the following explanation may shed some light on how the G159D mutation affects the dynamics of myofilament activation. The location of the mutant in the C-domain of cTnC suggests that it is unlikely to have a direct impact on $\mathrm{Ca}^{2+}$-mediated regulatory steps. One possible mechanism by which a mutation in the $\mathrm{C}$ domain of cTnC may affect $\mathrm{XB}$ recruitment dynamics is by altering the dynamics of XB-mediated effects on RU. Because part of the C-domain (adjacent to G159D) is in close contact with the IT arm of cardiac Tn $[13,37]$, it is possible that this mutation affects the global configuration of the IT arm in such a way that the status of RU is affected. The IT arm is formed by several key interactions, including that between $\mathrm{cTnT}$ and $\mathrm{cTnI}$ and hydrophobic interactions between $\mathrm{cTnI}$ and the C-domain of cTnC. Thus, it is reasonable to postulate that the IT arm of the troponin complex is affected by the G159D mutation.

Our study adds the following new data to our existing knowledge on the L29Q and G159D mutant-cardiac phenotype relationships. The L29Q mutation caused a small decrease in myofilament $\mathrm{Ca}^{2+}$ sensitivity, which was linked to an increase in RU off rate. Such an effect can be linked to the cardiac phenotype (i.e., hypertrophy) in L29Q proband by considering the fact that cardiac muscle cells operate under a very narrow range of physiological $\left[\mathrm{Ca}^{2+}\right]_{\text {free }}$. Therefore, any significant decrease in myofilament $\mathrm{Ca}^{2+}$ sensitivity may cause a significant decrease in tension at that given $\left[\mathrm{Ca}^{2+}\right]_{\text {free }}$. Thus, the hypertrophy associated with the L29Q substitution may be a compensatory response to overcome a decrease in tension caused by an attenuation of myofilament $\mathrm{Ca}^{2+}$ sensitivity. On the other hand, G159D mutation resulted in an increase in the XB cycling rate that is, $f$ and $g$, with no effects on maximal tension and $\mathrm{Ca}^{2+}$ sensitivity. Such increases in the $\mathrm{XB}$ cycling rate causes an increase in the cost of tension maintenance, subjecting the heart to a chronic stress to meet this increased energy demand. This functional consequence may be an impetus for the ventricular dilatation associated with the G159D mutation.

\section{Acknowledgments}

The authors thank Lindsey Muir for generating the cardiac TnC mutants. This work was supported by National Heart, Lung, and Blood Institute Grant no. R01-HL-075643 (to Murali Chandra).

\section{References}

[1] B. Hoffmann, H. Schmidt-Traub, A. Perrot, K. J. Osterziel, and R. Gessner, "First mutation in cardiac troponin C, L29Q, in a patient with hypertrophic cardiomyopathy," Human Mutation, vol. 17, no. 6, p. 524, 2001.

[2] A. P. Landstrom, M. S. Parvatiyar, J. R. Pinto et al., "Molecular and functional characterization of novel hypertrophic 
cardiomyopathy susceptibility mutations in TNNC1-encoded troponin C," Journal of Molecular and Cellular Cardiology, vol. 45, no. 2, pp. 281-288, 2008.

[3] W. K. Chung, C. Kitner, and B. J. Maron, "Novel frameshift mutation in Troponin C (TNNC1) associated with hypertrophic cardiomyopathy and sudden death," Cardiology in the Young, vol. 21, no. 3, pp. 345-348, 2011.

[4] C. C. Lim, H. Yang, M. Yang et al., "A novel mutant cardiac troponin $\mathrm{C}$ disrupts molecular motions critical for calcium binding affinity and cardiomyocyte contractility," Biophysical Journal, vol. 94, no. 9, pp. 3577-3589, 2008.

[5] J. Mogensen, R. T. Murphy, T. Shaw et al., "Severe disease expression of cardiac troponin $\mathrm{C}$ and $\mathrm{T}$ mutations in patients with idiopathic dilated cardiomyopathy," Journal of the American College of Cardiology, vol. 44, no. 10, pp. 2033-2040, 2004.

[6] R. E. Hershberger, N. Norton, A. Morales, D. Li, J. D. Siegfried, and J. Gonzalez-Quintana, "Coding sequence rare variants identified in MYBPC3, MYH6, TPM1, TNNC1, and TNNI3 from 312 patients with familial or idiopathic dilated cardiomyopathy," Circulation, vol. 3, no. 2, pp. 155-161, 2010.

[7] K. B. Campbell, M. V. Razumova, R. D. Kirkpatrick, and B. K. Slinker, "Nonlinear myofilament regulatory processes affect frequency-dependent muscle fiber stiffness," Biophysical Journal, vol. 81, no. 4, pp. 2278-2296, 2001.

[8] K. B. Campbell, M. V. Razumova, R. D. Kirkpatrick, and B. K. Slinker, "Myofilament kinetics in isometric twitch dynamics," Annals of Biomedical Engineering, vol. 29, no. 5, pp. 384-405, 2001.

[9] T. E. Gillis, C. R. Marshall, and G. F. Tibbits, "Functional and evolutionary relationships of troponin C," Physiological Genomics, vol. 32, no. 1, pp. 16-27, 2007.

[10] B. Liang, F. Chung, Y. Qu et al., "Familial hypertrophic cardiomyopathy-related cardiac troponin C mutation L29Q affects $\mathrm{Ca}^{2+}$ binding and myofilament contractility," Physiological Genomics, vol. 33, no. 2, pp. 257-266, 2008.

[11] A. Schmidtmann, C. Lindow, S. Villard et al., "Cardiac troponin C-L29Q, related to hypertrophic cardiomyopathy, hinders the transduction of the protein kinase A dependent phosphorylation signal from cardiac troponin I to C," FEBS Journal, vol. 272, no. 23, pp. 6087-6097, 2005.

[12] J. D. Potter, Z. Sheng, B. S. Pan, and J. Zhao, "A direct regulatory role for troponin $\mathrm{T}$ and a dual role for troponin $\mathrm{C}$ in the $\mathrm{Ca}^{2+}$ regulation of muscle contraction," Journal of Biological Chemistry, vol. 270, no. 6, pp. 2557-2562, 1995.

[13] S. Takeda, A. Yamashita, K. Maeda, and Y. Maéda, "Structure of the core domain of human cardiac troponin in the $\mathrm{Ca}^{2+}$ saturated form," Nature, vol. 424, no. 6944, pp. 35-41, 2003.

[14] D. Dweck, N. Hus, and J. D. Potter, "Challenging current paradigms related to cardiomyopathies: are changes in the $\mathrm{Ca}^{2+}$ sensitivity of myofilaments containing cardiac troponin C mutations (G159D and L29Q) good predictors of the phenotypic outcomes?" Journal of Biological Chemistry, vol. 283, no. 48, pp. 33119-33128, 2008.

[15] A. Neulen, R. Stehle, and G. Pfitzer, "The cardiac troponin C mutation Leu29Gln found in a patient with hypertrophic cardiomyopathy does not alter contractile parameters in skinned murine myocardium," Basic Research in Cardiology, vol. 104, no. 6, pp. 751-760, 2009.

[16] E. C. Dyer, A. M. Jacques, A. C. Hoskins et al., "Functional analysis of a unique troponin $\mathrm{c}$ mutation, gly159asp, that causes Familial dilated cardiomyopathy, studied in explanted heart muscle," Circulation, vol. 2, no. 5, pp. 456-464, 2009.
[17] M. Mirza, S. Marston, R. Willott et al., "Dilated cardiomyopathy mutations in three thin filament regulatory proteins result in a common functional phenotype," Journal of Biological Chemistry, vol. 280, no. 31, pp. 28498-28506, 2005.

[18] P. Robinson, P. J. Griffiths, H. Watkins, and C. S. Redwood, "Dilated and hypertrophic cardiomyopathy mutations in troponin and $\alpha$-tropomyosin have opposing effects on the calcium affinity of cardiac thin filaments," Circulation Research, vol. 101, no. 12, pp. 1266-1273, 2007.

[19] B. J. Biesiadecki, T. Kobayashi, J. S. Walker, R. J. Solaro, and P. P. de Tombe, "The troponin C G159D mutation blunts myofilament desensitization induced by troponin I Ser23/24 phosphorylation," Circulation Research, vol. 100, no. 10, pp. 1486-1493, 2007.

[20] L. C. Preston, C. C. Ashley, and C. S. Redwood, "DCM troponin C mutant Gly159Asp blunts the response to troponin phosphorylation," Biochemical and Biophysical Research Communications, vol. 360, no. 1, pp. 27-32, 2007.

[21] M. Chandra, M. L. Tschirgi, S. J. Ford, B. K. Slinker, and K. B. Campbell, "Interaction between myosin heavy chain and troponin isoforms modulate cardiac myofiber contractile dynamics," American Journal of Physiology, vol. 293, no. 4, pp. R1595-R1607, 2007.

[22] M. Chandra, M. L. Tschirgi, I. Rajapakse, and K. B. Campbell, "Troponin T modulates sarcomere length-dependent recruitment of cross-bridges in cardiac muscle," Biophysical Journal, vol. 90, no. 8, pp. 2867-2876, 2006.

[23] M. Chandra, V. L. M. Rundell, J. C. Tardiff, L. A. Leinwand, P. P. De Tombe, and R. J. Solaro, " $\mathrm{Ca}^{2+}$ activation of myofilaments from transgenic mouse hearts expressing R92Q mutant cardiac troponin T," American Journal of Physiology, vol. 280, no. 2, pp. H705-H713, 2001.

[24] X. Guo, J. Wattanapermpool, K. A. Palmiter, A. M. Murphy, and R. J. Solaro, "Mutagenesis of cardiac troponin I. Role of the unique NH2-terminal peptide in myofilament activation," Journal of Biological Chemistry, vol. 269, no. 21, pp. 1521015216, 1994.

[25] B. S. Pan and R. G. Johnson, "Interaction of cardiotonic thiadiazinone derivatives with cardiac troponin C," Journal of Biological Chemistry, vol. 271, no. 2, pp. 817-823, 1996.

[26] D. E. Montgomery, J. C. Tardiff, and M. Chandra, "Cardiac troponin T mutations: correlation between the type of mutation and the nature of myofilament dysfunction in transgenic mice," Journal of Physiology, vol. 536, no. 2, pp. 583-592, 2001.

[27] J. C. Tardiff, S. M. Factor, B. D. Tompkins et al., "A truncated cardiac troponin $\mathrm{T}$ molecule in transgenic mice suggests multiple cellular mechanisms for familial hypertrophic cardiomyopathy," Journal of Clinical Investigation, vol. 101, no. 12, pp. 2800-2811, 1998.

[28] M. Chandra, M. L. Tschirgi, and J. C. Tardiff, "Increase in tension-dependent ATP consumption induced by cardiac troponin T mutation," American Journal of Physiology, vol. 289, no. 5, pp. H2112-H2119, 2005.

[29] M. Chandra, D. E. Montgomery, J. J. Kim, and R. J. Solaro, "The N-terminal region of troponin $\mathrm{T}$ is essential for the maximal activation of rat cardiac myofilaments," Journal of Molecular and Cellular Cardiology, vol. 31, no. 4, pp. 867-880, 1999.

[30] M. Chandra, J. J. Kim, and R. J. Solaro, “An improved method for exchanging troponin subunits in detergent skinned rat cardiac fiber bundles," Biochemical and Biophysical Research Communications, vol. 263, no. 1, pp. 219-223, 1999. 
[31] A. Fabiato and F. Fabiato, "Calculator programs for computing the composition of the solutions containing multiple metals and ligands used for experiments in skinned muscle cells," Journal de Physiologie, vol. 75, no. 5, pp. 463-505, 1979.

[32] R. L. Lieber, Y. Yeh, and R. J. Baskin, "Sarcomere length determination using laser diffraction. Effect of beam and fiber diameter," Biophysical Journal, vol. 45, no. 5, pp. 1007-1016, 1984.

[33] P. P. de Tombe and G. J. M. Stienen, "Protein kinase A does not alter economy of force maintenance in skinned rat cardiac trabeculae," Circulation Research, vol. 76, no. 5, pp. 734-741, 1995.

[34] G. J. M. Stienen, G. Zaremba, and G. Elzinga, "ATP utilization for calcium uptake and force production in skinned muscle fibres of Xenopus laevis," Journal of Physiology, vol. 482, no. 1, pp. 109-122, 1995.

[35] K. B. Campbell, M. Chandra, R. D. Kirkpatrick, B. K. Slinker, and W. C. Hunter, "Interpreting cardiac muscle force-length dynamics using a novel functional model," American Journal of Physiology, vol. 286, no. 4, pp. H1535-H1545, 2004.

[36] K. Campbell, "Rate constant of muscle force redevelopment reflects cooperative activation as well as cross-bridge kinetics," Biophysical Journal, vol. 72, no. 1, pp. 254-262, 1997.

[37] M. V. Vinogradova, D. B. Stone, G. G. Malanina et al., " $\mathrm{Ca}^{2+}$ regulated structural changes in troponin," Proceedings of the National Academy of Sciences of the United States of America, vol. 102, no. 14, pp. 5038-5043, 2005. 

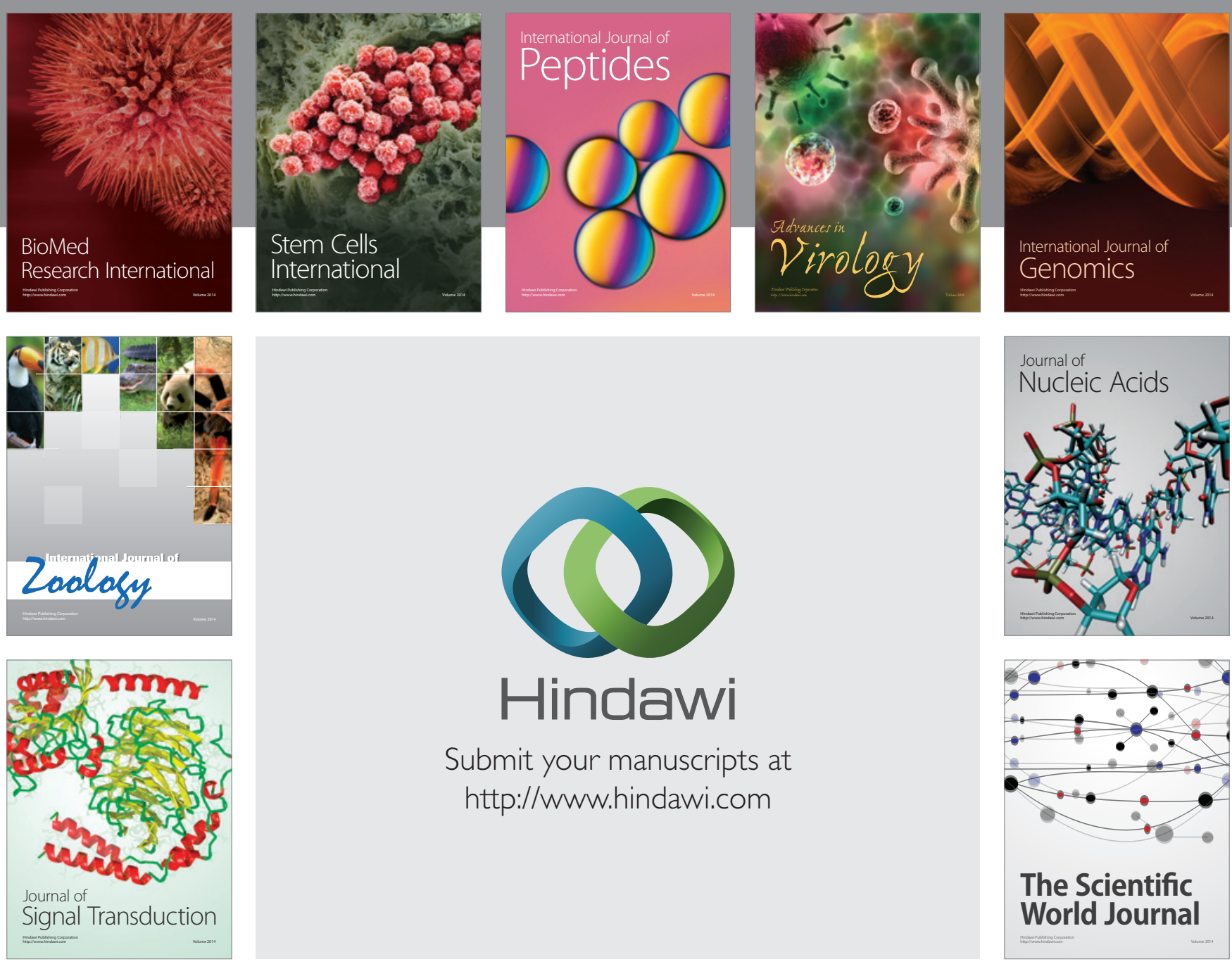

Submit your manuscripts at

http://www.hindawi.com
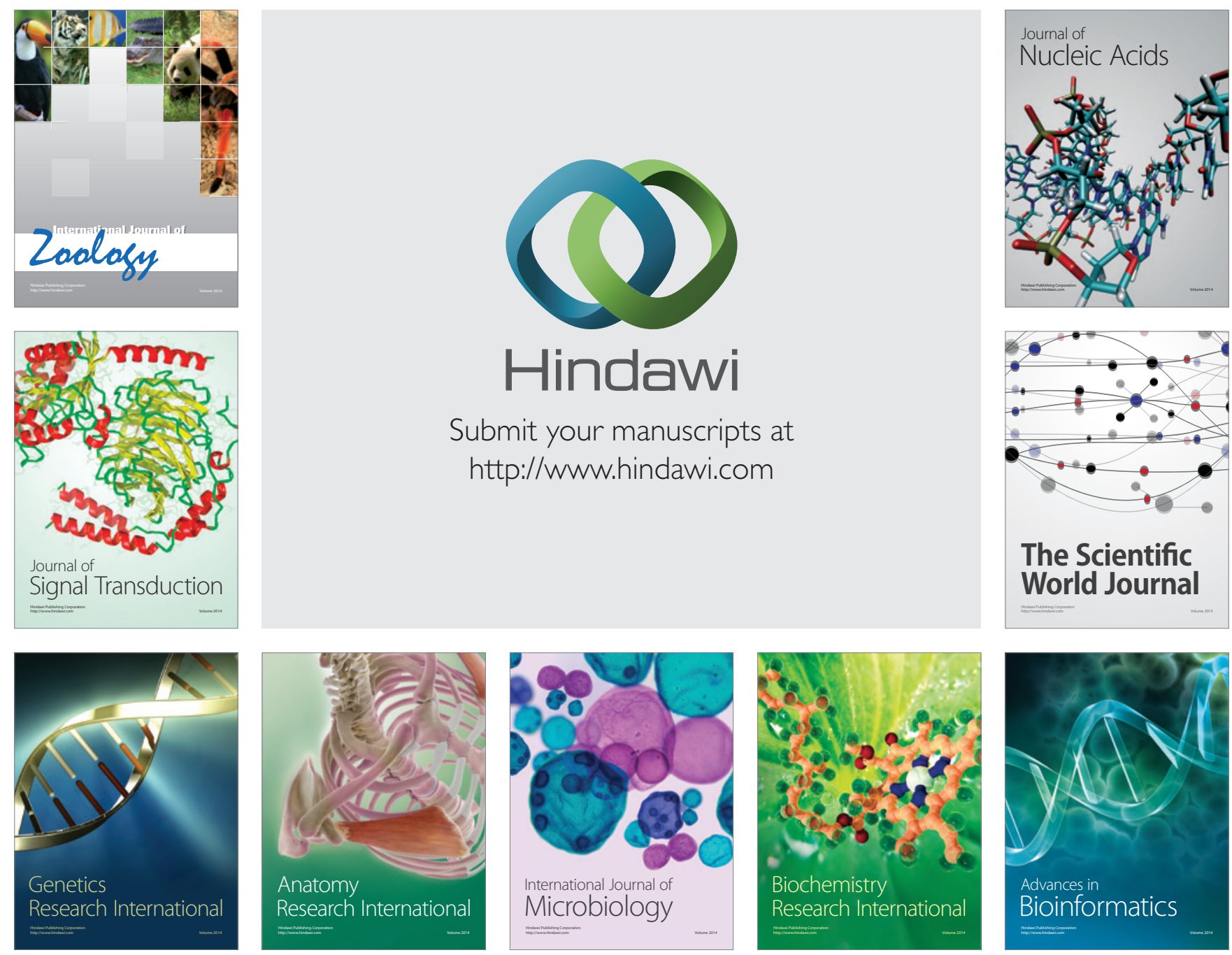

The Scientific World Journal
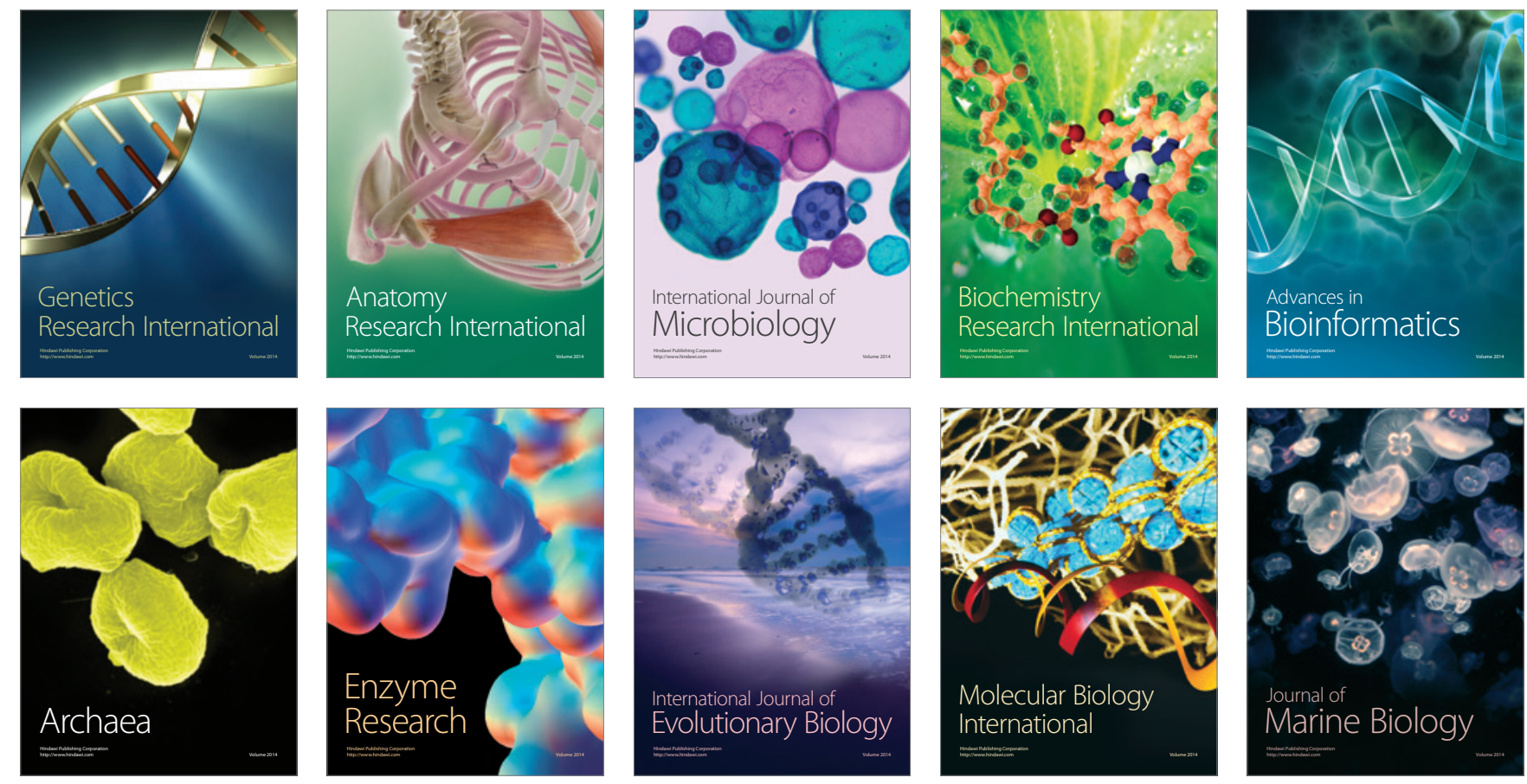